\section{MS17-P12 Neutron diffraction study of piezoelectric material under electric field}

Takuro Kawasaki ${ }^{1}$, Takayoshi Ito ${ }^{2}$, Yasuhiro Inamura ${ }^{1}$, Takeshi Nakatani $^{1}$, Stefanus Harjo ${ }^{1}$, Wu Gong ${ }^{1}$, Kazuya Aizawa ${ }^{1}$, Takaaki Iwahashi ${ }^{1}$

\section{J-PARC Center, Japan Atomic Energy Agency}

2. Technical Support Group, CROSS-Tokai

email: takuro.kawasaki@j-parc.jp

Piezoelectric material is widely used as an actuator and other various devices because the size of the material can be controlled by the applied electric field. Two types of microscopic deformation, lattice strain and domain switching, produces this macroscopic deformation. On the other hand, time-resolved measurement is attracting great interest in order to observe transient phenomena of materials under external fields. The behaviors of the lattice and the domain under the cyclic electric field can be studied by time-resolved in-situ diffraction experiment.In this study, the lattice strain and the domain characteristic of piezoelectric material under static and cyclic electric fields were studied by time-of-flight neutron diffraction. The diffraction intensities from PZT-based ceramics were measured at Engineering Materials Diffractometer TAKUMI in J-PARC. The intensities in the parallel direction and the vertical direction to the field were measured simultaneously by $\pm 90^{\circ}$ detector banks. The collected data were divided based on the voltage and the phase conditions of applied electric field and were converted to diffraction patterns using the data reduction software developed in J-PARC. The variation of the position and the intensity of the diffraction peaks according to the applied field were found in the patterns. The responses of the lattice and the domain to the field were successfully observed.

Keywords: neutron diffraction, piezoelectric material, lattice strain

\author{
MS17-P13 High-pressure study of \\ $\mathrm{Mn}\left(\mathrm{BH}_{4}\right)_{2}$ : a new polymorphs with high \\ hydrogen density \\ Nikolay Tumanov ${ }^{1}$, Elsa Roedern ${ }^{2}$, Dorrit B. Nielsen ${ }^{2}$, Torben R. \\ Jensen ${ }^{2}$, Alexander V. Talyzin ${ }^{3}$, Radovan Černý ${ }^{4}$, Dmitry \\ Chernyshov $^{5}$, Vladimir Dmitriev ${ }^{5}$, Zbigniew Łodziana ${ }^{6}$, Yaroslav \\ Filinchuk $^{1}$
}

1. Institute of Condensed Matter and Nanosciences, Université catholique de Louvain, place L. Pasteur 1, 1348 Louvain-la-Neuve, Belgium

2. Center for Materials Crystallography, Interdisciplinary Nanoscience Center (iNANO) and Department of Chemistry, University of Aarhus, Langelandsgade 140, DK-8000 Aarhus C, Denmark

3. Department of Physics, Linnaeus väg 24, Umeå University, Umeå S-90187, Sweden

4. Laboratory of Crystallography, DPMC-MaNEP, University of Geneva, quai Ernest-Ansermet 24, 1211 Geneva, Switzerland

5. Swiss Norwegian Beam Lines (SNBL) at the European Synchrotron Radiation Facility (ESRF), rue Jules Horowitz, 38043 Grenoble, France

6. INP Polish Academy of Sciences, Department of Structural Research, ul. Radzikowskiego 152, 31-342 Kraków, Poland

email: nikolay.tumanov@uclouvain.be

Hydrogen economy is one of the perspective trends in the future of renewable energy, but hydrogen storage is still a limiting factor. A possible way is to chemically bound hydrogen in light-weight compounds. In this context, metal borohydrides have been studied intensively in recent years. One of the problems in the practical application of borohydrides as hydrogen storage materials is often a too high or a too low decomposition temperature, but manganese borohydride is one of the borohydrides with moderate temperature of decomposition $\left(160{ }^{\circ} \mathrm{C}\right)$. The structure and properties of metal borohydrides are usually studied at ambient conditions, but applying high hydrostatic pressure to the compounds not only adds an additional dimension to the phase diagram, revealing new high-pressure polymorphs of borohydrides, but may also allow to discover new high-pressure phases that are stable upon decompression. Such compounds are not only denser than those known to form at ambient conditions, but may also provide new or improved properties to a system, e.g. regarding, stability, decomposition and melting temperatures, etc. For instance, $\delta-\mathrm{Mg}\left(\mathrm{BH}_{4}\right)_{2}$, obtained by compression of $\mathrm{Mg}\left(\mathrm{BH}_{4}\right)_{2}$ to $\sim \mathrm{GPa}^{4}$, is more stable in air [1], than its ambient pressure phases.

The high-pressure behavior of $\mathrm{Mn}\left(\mathrm{BH}_{4}\right)_{2}$ was studied up to $15 \mathrm{GPa}$ using diamond anvil cells and powder X-ray diffraction and Raman spectroscopy and two new polymorphs was discovered using combination of diffraction and DFT calculations. The first polymorph, $\delta-\mathrm{Mn}\left(\mathrm{BH}_{4}\right)_{2}$, forms near $1 \mathrm{GPa}$ and is isostructural to a magnesium analog, $\delta-\operatorname{Mg}\left(\mathrm{BH}_{4}\right)_{2}$. This polymorph is stable upon decompression to ambient conditions. The same polymorph was obtained by compression of $\alpha-\mathrm{Mn}\left(\mathrm{BH}_{4}\right)_{2}$ in a large-volume steel press, as well as by high-energy ball milling. The thermal stability was studied by in situ powder X-ray diffraction and thermal analysis coupled with mass-spectroscopy. $\delta-\mathrm{Mn}\left(\mathrm{BH}_{4}\right)$ transforms back to $\alpha-\mathrm{Mn}\left(\mathrm{BH}_{4}\right)$, upon heating $\left(67-109^{\circ} \mathrm{C}\right)$ and decomposition is initiated at $130{ }^{\circ} \mathrm{C}$, releasing hydrogen and some diborane. The high-pressure polymorph, $\delta-\mathrm{Mn}\left(\mathrm{BH}_{4}\right)_{2}$, transforms into a second, $\delta$ '- $\mathrm{Mn}\left(\mathrm{BH}_{4}\right)_{2}$, in the pressure range of 8.6-11.8 GPa, with 
$\mathrm{BH}_{4}^{-}$groups ordered in a superstructure. $\delta^{\prime}-\mathrm{Mn}\left(\mathrm{BH}_{4}\right)_{2}$ is not isostructural to the second high-pressure phase of $\operatorname{Mg}\left(\mathrm{BH}_{4}\right)_{\text {. Equations of state were determined for } \alpha-\text { and }}$ $\delta-\mathrm{Mn}\left(\mathrm{BH}_{4}^{2}\right)_{2}$

Y. Filinchuk, B. Richter, T. R. Jensen, V. Dmitriev, D. Chernyshov, and H. Hagemann, Angew. Chem. Int. Ed., 2011, 50, 11162.

Keywords: hydrogen storage, borohydrides
MS17-P14 In situ X-ray crystallography of colloidal crystals under sintering conditions

Alexey V. Zozulya ${ }^{1}$, Elena A. Sulyanova ${ }^{2}$, Anatoly Shabalin ${ }^{1}$, Janne-Mieke Meijer ${ }^{3}$, Dmitry Dzhigaev ${ }^{1,4}$, Oleg Gorobtsov ${ }^{1,5}$, Ruslan P. Kurta ${ }^{6}$, Sergey Lazarev ${ }^{1}$, Ivan Zaluzhnyy ${ }^{1,4}$, Ulf Lorenz $^{7}$, Andrej Singer ${ }^{8}$, Oleksandr Yefanov ${ }^{9}$, Ilya Besedin ${ }^{1,4}$, Michael Sprung ${ }^{1}$, Andrei V. Petukhov ${ }^{3}$, Ivan A. Vartanyants ${ }^{1}$

1. Deutsches Elektronen-Synchrotron DESY Hamburg

2. Shubnikov Institute of Crystallography RAS, Leninskii pr. 59, 119333 Moscow, Russia

3. van 't Hoff laboratory for Physical and Colloid Chemistry, Debye Institute for Nanomaterials Science, University of Utrecht, Padualaan 8, 3508 TB Utrecht, The Netherlands

4. National Research Nuclear University MEPhI (Moscow Engineering Physics Institute), Kashirskoye ch. 31, 115409 Moscow, Russia

5. NRC Kurchatov Institute, Akademika Kurchatova pl. 1, 123182 Moscow, Russia

6. European XFEL GmbH, Notkestraße 85, D-22607 Hamburg, Germany

7. Department of Chemistry, University of Potsdam, D-14476 Potsdam, Germany

8. University of California, 9500 Gilman Dr., La Jolla, San Diego, California 92093, USA

9. Center for Free-Electron Laser Science, DESY, Notkestraße 85, D-22607 Hamburg, Germany

email: alexey.zozulya@desy.de

Periodic photonic nanostructures enable to manipulate and control light emission and propagation due to existence of band gaps $[1,2]$. Photonic crystals represent a new class of nanomaterials with promising applications in optical communication lines, sensor technology and data storage. Colloidal crystals formed by self-assembly of sub-micrometer colloidal spherical particles [3] are especially attractive due to their large area, low fabrication costs and a wide range of particle size and shape. Investigation of a real structure of these materials and its temperature dependence is of utmost importance for the future development of photonic devices.

Band gap properties of colloidal crystals can be altered by thermal treatment (dry sintering) [4]. The physical reason for this phenomenon is not well understood. The behavior of low-order spin-coated polystyrene (PS) colloidal films under annealing treatment provides only rough picture of structural evolution [5]. Our recent studies of high-quality PS colloidal crystals upon incremental heating conditions have revealed detailed scenario of colloidal crystal melting $[6,7]$. X-ray diffraction studies of PS colloidal crystals have been carried out in transmission and reflection geometries using high resolution X-ray scattering setup at the Coherence Beamline P10 of the PETRA III light source. Diffraction peak parameters, such as q-values, integrated peak intensities, the radial and azimuthal widths, were analyzed as a function of temperature [7]. Temperature dependencies of lattice distortions, mosaic spread and a size of coherently scattering domain were evaluated by Williamson-Hall method. As a result we identified four stages of structural evolution in PS crystals upon sintering: steady-state, pre-melting, shape transformation and crystal melting. The observed peculiarities of lattice distortions indicate that the evolution of in-plane and out-of-plane order plays a central role in the process of colloidal crystal melting.

\section{References}

